\title{
The hydrothermal decomposition of biomass and waste to produce bio-oil
}

\author{
P. De Filippis, B. de Caprariis, M. Scarsella \& N. Verdone \\ Chemical Engineering Department, Sapienza University of Rome, Italy
}

\begin{abstract}
The hydrothermal decomposition of biomass is an alternative to the traditional pyrolysis processes to obtain liquid fuels with an increased energy density. Hydrothermal liquefaction is claimed to produce bio-oils with improved characteristics such as a reduced content of oxygen, avoiding at the same time the energy consuming biomass drying step necessary in the traditional thermochemical processes. However, at present the use of this technology is limited by the severe conditions needed and by the difficulties connected with the realization of a continuous process. In this work an innovative process, operating at atmospheric pressure and at moderate temperatures, is proposed, where water subcritical conditions are achieved locally by means of the energy released during cavitation. The process was tested on lab-scale on two biomass feeds obtaining a crude bio-oil with a composition very similar to that of fossil oil and greatly improved respect to those obtained in traditional pyrolysis processes.
\end{abstract}

Keywords: hydrothermal pyrolysis, biomass, biofuels.

\section{Introduction}

The increasing energy demand is drawing the attention to raw materials alternative to fossil fuels, encouraging the development of processes and technologies for the valorisation of alternative sources of energy such as biomass and waste. The main issue related to the use of these materials is represented by their low energy density and high water content, which, in the case of biomass, can reach up to $40 \%$ in weight. Thermochemical processes such as pyrolysis and gasification are attractive solutions to produce gaseous and liquid fuels with increased energy density [1]. However, these traditional technologies present at 
least two critical issues: the need of dry feedstock to achieve a positive energy balance and the need of a secondary process to upgrade the raw products to commercially valuable fuels.

To overcome these limitations, hydrothermal liquefaction (HTL) treatments can represent an alternative to convert biomass and waste into liquid products. In a HTL process, the biomass macromolecules are first depolymerized into light molecules and then the obtained unstable fraction is repolymerized into oil compounds [2]. Water is used as reaction medium and thus the organic feeds can be directly processed avoiding the preliminary energy consuming drying step. Hydrothermal decomposition is therefore well suited to process high and medium moisture ( $>20 \%$ moisture) feedstocks.

During the process, a mixture of solid fuel and water is heated to $280-370^{\circ} \mathrm{C}$ under a pressure ranging from 10 to $25 \mathrm{MPa}$ [3]. In these subcritical conditions, water changes its physical and chemical properties, acting both as reactant and catalyst. In this way the feed can be directly converted into crude oil [4], which in turn is easily separated from water due to its low oxygen content. This results into an energy content higher than pyrolysis-derived oils obtained without upgrading processes [2]. Oxygen removal occurs mostly by dehydration, which removes oxygen in the form of water, and by decarboxylation, which removes oxygen in the form of carbon dioxide [2].

The majority of the hydrothermal liquefaction technologies developed from the late ' 70 s to the present had limited success, generally achieving the production of thick and thermally unstable liquid products that needed expensive refining and upgrading treatments to obtain a product in the range of diesel fuel [2]. This, together with the need to operate at severe conditions, the difficulties connected with the realization of a continuous process, the low liquid yield due to the limited dry matter content in the feed and to the low conversion rates, hampered the commercialization of these technologies and the development of economically viable processes and industrial size plants.

In literature the hydrothermal liquefaction of biomass has been investigated in many studies $[5,6]$. Singh et al. analysed the hydrothermal conversion of wheat husk using an alkaline catalyst, obtaining a bio-oil yield of $31 \%$. The effect of alkaline catalyst seems to give improved conversions [7]. From the liquefaction of swichgrass, Ranmsurn and Gupta obtained a bio-crude with a low oxygen content which led to a calorif value of the bio-crude of $27 \mathrm{MJ} / \mathrm{kg}$ [8].

To overcome the problems related with the necessity to operate in severe conditions, in this work an innovative process is proposed. The water subcritical conditions are achieved locally by means of the energy released during cavitation. In fact, during cavitation in a liquid medium bubbles are formed and can collapse violently, generating high temperature (about 10,000 K) and high pressure (about $100 \mathrm{MPa}$ ) conditions, which can induce the same chemical and physical transformations of HTL [9].

In this work a homogenizer with an optimized geometry is used to induce cavitation inside a reactor held at $250^{\circ} \mathrm{C}$ and 1 atm. A liquid product characterized by a fossil fuel-like elemental composition is obtained from wet biomass and waste. 


\section{Experimental}

\subsection{Experimental set-up}

The reactor consists in a two neck round-bottom flask in which is placed the homogenizer. The homogenizer consists of an impeller which forces the material to pass through a series of fissures, inducing the cavitation. This particular geometry and the high rotational speed (up to 20,000 rpm) of the homogenizer are fundamental to obtain the cavitation needed to reach HTL conditions. The reactor was loaded with a mixture of naphthenic based oil and biomass. The naphthenic based oil (Nyflex 222, Nynas, $27^{\circ} \mathrm{API}$ ), which has the function of liquid medium, was chosen for its high thermal stability (decomposition temperature $>300^{\circ} \mathrm{C}$ ), to avoid its decomposition during the process. A K-type thermocouple was used to control the temperature inside the reactor, heated by a heating mantle. The homogenizer was switched on when the temperature reached the operative value and then having it kept constant during the one hour tests.

In Table 1 the operative conditions used during the experiments are reported. The mass ratio between the oil and the solid fuel was kept constant at about 3 for all the experiments. Experimental tests at different temperatures were performed in order to study the influence of the bulk temperature on the crude oil yields. $\mathrm{CaO}$ was used as catalyst in a mass ratio with respect to the feedstock of 0.05 .

A laboratory distillation set-up was used to condense the liquid products which were recovered in a flask.

Table 1: Operative conditions used during the experiments.

\begin{tabular}{lc}
\hline Naphthenic based Oil $(\mathrm{g})$ & 70 \\
Biomass $(\mathrm{g})$ & 21 \\
Rotational speed $(\mathrm{RPM})$ & 20000 \\
$\mathrm{CaO}(\mathrm{g})$ & 1 \\
Temperature $\left({ }^{\circ} \mathrm{C}\right)$ & $250 ; 280$ \\
\hline
\end{tabular}

\subsection{Materials}

Two different feedstocks were used in the experimental tests, sawdust and poultry manure. The physical and chemical properties of the two materials are reported in Table 2. It can be noticed that the water content, higher than $20 \%$, satisfies the minimum value needed for hydrothermal liquefaction.

\section{Results and discussions}

In Table 3 the list of all the experimental tests is reported. To confirm that the naphthenic oil used as medium does not decompose to lighter fractions, blank tests were performed on the oil and on its mixture with a water amount similar to that of the solid biomass $(20 \%)$. The results, reported in Table 4, show that the 
oil does not decompose. In the Test 2 , in fact, the recovered liquid was composed of water with a small quantity of entrained oil, while during the Test 1 no liquid was recovered.

To demonstrate that the system proposed is able to originate locally HTL conditions, the results were compared with those obtained in an experimental test (Test 8) conducted using a traditional mechanical stirrer which should not induce cavitation. In this last case the recovered liquid, produced during the reaction, is composed of the water contained in the feed and of a small quantity of bio-oil ( $2 \% \mathrm{wt}$. of the feed) produced by the beginning of thermal pyrolysis.

In Table 4 the results of all the experimental tests are reported. It can be noticed from the comparison between Test 3 and 5 that the presence of the catalyst has an influence on the oil yield, which increases of $40 \%$.

Table 2: $\quad$ Physical and chemical characteristics of the selected feeds.

\begin{tabular}{ccc}
\hline Proximate analysis & Sawdust & Poultry manure \\
\hline Moisture & 27.0 & 26.0 \\
Volatile substance (\% dry basis) & 67.0 & 61.0 \\
Fixed carbon (\% dry basis) & 22.0 & 15.0 \\
Ash (\% dry basis) & 11.0 & 24.0 \\
Size (mm) & $<1.0$ & $<3.0$ \\
\hline Elemental analysis (\% dry basis) & & \\
\hline Carbon & 46.3 & 39.3 \\
Hydrogen & 5.4 & 4.6 \\
Nitrogen & 0.6 & 3.1 \\
Oxygen & 36.6 & 28.0 \\
Sulphur & 0.1 & 0.0 \\
\hline
\end{tabular}

Table 3: List of the experimental tests.

\begin{tabular}{llll}
\hline & $\mathrm{T}\left({ }^{\circ} \mathrm{C}\right)$ & $\mathrm{CaO}(\mathrm{g})$ & Feed \\
\hline Test 1 blank (only oil) & 250 & 1 & No \\
Test 2 blank (oil + 20\% water) & 250 & 1 & No \\
Test 3 & 250 & 1 & Poultry manure \\
Test 4 & 280 & 1 & Poultry manure \\
Test 5 & 250 & 0 & Poultry manure \\
Test 6 & 250 & 1 & Sawdust \\
Test 7 & 280 & 1 & Sawdust \\
Test 8 (mechanical stirrer) & 280 & 1 & Poultry manure \\
\hline
\end{tabular}


Table 4: Results of the experimental tests.

\begin{tabular}{lllll}
\hline & \% water & \% oil & \% gas & \% residue \\
\hline Test 1 & 0 & 0 & 0 & 100 \\
Test 2 & 20 & 1 & 0 & 79 \\
Test 3 & 32 & 14 & 11 & 43 \\
Test 4 & 29 & 19 & 7 & 35 \\
Test 5 & 31 & 10 & 11 & 48 \\
Test 6 & 34 & 17 & 8 & 41 \\
Test 7 & 33 & 21 & 9 & 37 \\
Test 8 & 26 & 2 & 3 & 69 \\
\hline
\end{tabular}

Higher temperatures (Tests 4 and 7) lead to an improving of bio-oil yield for both the tested feeds. In Figure 1 the TBP curve of the poultry manure oil fraction is reported, being very similar to that of a fossil gasoil fraction. The figure shows that the product is characterized by a high final boiling point probably caused by the entrainment of a small amount of Niflex oil with the vapour flow.

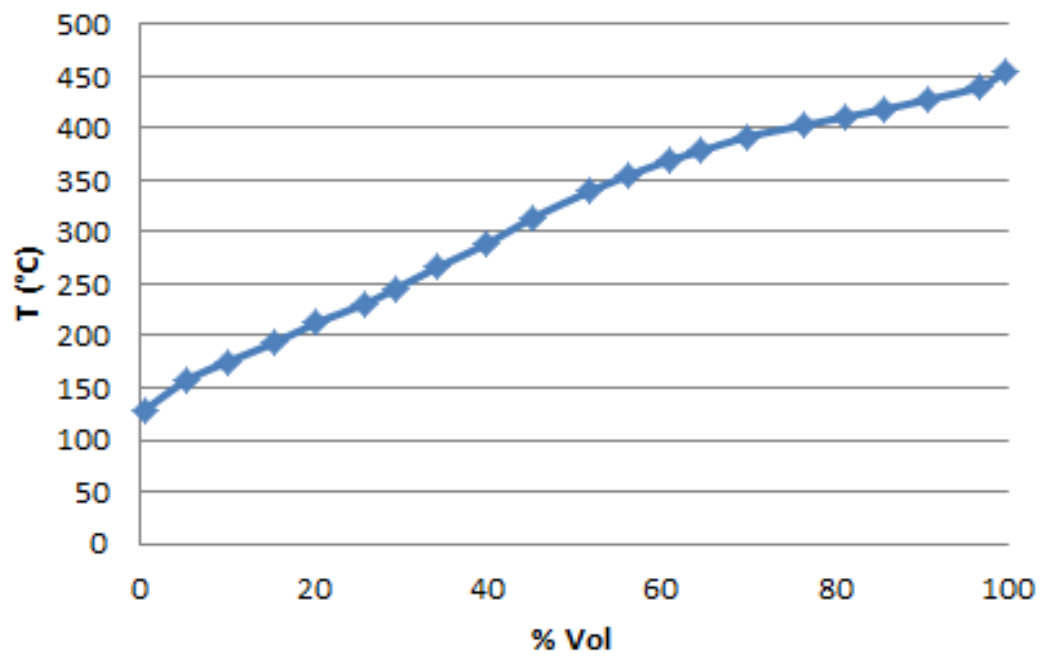

Figure 1: $\quad$ TPB curve of the poultry manure crude oil.

The elemental analysis of the two obtained bio-oils was performed and the results were compared with those of a bio-oil obtained from a traditional wood pyrolysis and of a fossil oil [10] (Table 5). The quality of the obtained bio-oils is considerably enhanced with respect to that obtained from traditional wood pyrolysis. The oxygen content is very low and the ratio $\mathrm{C} / \mathrm{H}$ is very similar to that of fossil oil. 
Table 5: Elemental composition of the bio-oils obtained with the cavitation process.

\begin{tabular}{lllll}
\hline & Poultry manure & Sawdust & Wood bio oil & Fossil oil \\
\hline$\% \mathrm{C}$ & 84.0 & 82.0 & 56.4 & $83.0-87.0$ \\
$\% \mathrm{H}$ & 12.5 & 12.6 & 6.2 & $10.0-14.0$ \\
$\% \mathrm{O}$ & 2.3 & 5.1 & 37.3 & $0.05-1.5$ \\
$\% \mathrm{~N}$ & 1.2 & 0.3 & 0.1 & $0.01-0.7$ \\
$\% \mathrm{~S}$ & 0 & 0 & 0 & $0.75-1.0$ \\
\hline
\end{tabular}

\section{Conclusions}

Hydrothermal liquefaction is an attractive alternative to traditional thermal treatments to produce crude bio-oil having properties similar to that of fossil fuels. The severe operative conditions needed for the process represent a limitation to the development of this technology. In this work an innovative method to reach the hydrothermal liquefaction conditions needed to decompose biomass at ambient pressure and moderate temperatures is proposed and good results are obtained. The energy necessary to break molecules is given by cavitation in which high temperature and pressure are locally reached. For the sawdust the oil yield exceeds $20 \%$ by weight when the process is carried at $280^{\circ} \mathrm{C}$. The obtained oil has a composition very similar to that of fossil oil. With respect to a bio-oil obtained from traditional pyrolysis, the oxygen is nearly removed in the case of poultry manure oil and lowered of $87 \%$ for the sawdust oil. Furthermore the $\mathrm{C} / \mathrm{H}$ ratio is very similar to that of fossil oil.

\section{References}

[1] Verdone, N., De Filippis, P., Scarsella, M., de Caprariis, B., Waste gasification in an up-draft fixed-bed gasifier: Experimental study and model validation. WIT Transactions on Ecology and the Environment, 163, pp. 113-122, 2012.

[2] Peterson. A.A., Vogel, F., Lachance, R.P., Froling, M., Antal, M. J., Tester, J.W., Thermochemical biofuel production in hydrothermal media: a review of sub- and supercritical water technologies. Energy \& Environmental Science, 1, pp. 32-65, 2008.

[3] Maki-Arvela, P., Kubickova, I., Snare, M., Eranen, K., Murzin, D. Y., Catalytic Deoxygenation of Fatty Acids and Their Derivatives. Energy \& Fuels, 21, pp. 30-41, 2007.

[4] Snare, M., Kubickova, I., Maki-Arvela, P., Eranen, K., Warna, J., Murzin, D. Y., Production of diesel fuel from renewable feeds: Kinetics of ethyl stearate decarboxylation. Chemical Engineering Journal, 134 (1-3), pp. 29-34, 2007.

[5] Elliott, D. C., Hart, T. R., Schmidt, A. J., Neuenschwander, G. G., Rotness, L. J. et al., Process development for hydrothermal liquefaction of 
algae feedstocks in a continuous-flow reactor. Algal Research, 2(4), pp. 445-454, 2013.

[6] Chen, W., Zhang, Y., Zhang, J., Yu, G., et al., Hydrothermal liquefaction of mixed-culture algal biomass from wastewater treatment system into bio-crude oil. Bioresource Technology, 152, pp.130-139, 2014.

[7] Singh, R., Bhaskar, T., Dora, S., Balagurumurthy, B., Catalytic hydrothermal upgradation of wheat husk. Biouresource Technology, 149, pp. 446-451, 2013.

[8] Ramsurn, H., Gupta, R. B., Deoxy-liquefaction of switchgrass in supercritical water with calcium formate as an in-situ hydrogen donor. Bioresource Technology, 143, pp. 575-583, 2013.

[9] Iskalieva, A., Yimmou, B. M., Gogate, P. R., Horvath, M., et al., Cavitation assisted delignification of wheat straw: A review. Ultrasonics Sonochemistry, 19(5), pp. 984-993, 2012.

[10] Miao, X., Wu, Q., Yang, C., Fast pyrolysis of microalgae to produce renewable fuels. Journal of Analytical and Applied Pyrolysis, 71, pp. 855-863, 2004. 